MATEC Web of Conferences 1, 07001 (2012)

DOI: $10.1051 /$ matecconf $/ 20120107001$

(C) Owned by the authors, published by EDP Sciences, 2012

\title{
Relation between synchronization of a ring of coupled Rössler systems and observability
}

\author{
Adrien Kerfourn ${ }^{1}$, Laurent Laval $^{2}$, and Christophe Letellier ${ }^{1, \mathrm{a}}$ \\ 1 CORIA - Unviversité de Rouen, BP 12, F-76801 Saint-Etienne du Rouvray cedex, France \\ 2 ENSEA, 6 avenue du Ponceau, 95014 Cergy-Pontoise cedex, France
}

\begin{abstract}
It is shown how a lack of observability associated with the coupling variable can affect the resulting dynamics of a ring of Rössler systems, here investigated in a state sub-space of a local oscillator.
\end{abstract}

From the seminal paper by Pecora and Carrol [1] on synchronization, it is known that the quality of synchronization is dependent on the variable chosen for coupling the systems. In most of the papers, the results are presented using one variable chosen a priori without any justification. Very frequently, systems are empirically coupled using the variable providing the best observability: for instance, Rössler systems are coupled using variable $y$ [3] which is the best variable since there is a global diffeomorphism between the original state space of the Rössler system and the differential embedding induced by variable $y$ and its first two derivatives [4]: it represents the best quality one can expect when a state space is reconstructed from a single scalar time series [4]. This variable is also the best one to use for synchronizing Rössler systems [2]. Lorenz systems are often coupled using variable $x$ [1]. Although there is no global diffeomorphism between the state space of the Lorenz system and the $x$-induced differential embedding, variable $x$ provides the best observability [5]. A variable is associated with a lack of observability when there is a domain - the observability singular manifold defined by Det $\mathcal{J}_{\Phi_{s}}=0$ where $\mathcal{J}_{\Phi_{s}}$ is the jacobian matrix of the coordinate transformation $\Phi$ between the original state space and the differential embedding induced by the "measured" variable $s-$ in the state space which cannot be properly reconstructed using the chosen variable. Obviously the location of the observability singular manifold related to the chaotic attractor has an impact on the quality of the variable.

The interplay between observability and synchronization was recently shown for bidirectional coupling between two nearly identical systems [2]: the range of values of the coupling parameter for which complete and/or phase synchronization can be obtained is the largest when the systems are coupled via a variable providing a good observability as shown, for instance, in the case of two Rössler systems bidirectionaly coupled (Fig. 1).

We here extend this approach to a ring of bidirectionnally coupled Rössler systems

$$
\left\{\begin{array}{l}
\dot{x}=\omega(-y-z) \\
\dot{y}=\omega(x+a y) \\
\dot{z}=\omega(b+z(x-c))
\end{array}\right.
$$

a e-mail: Christophe.Letellier@coria.fr

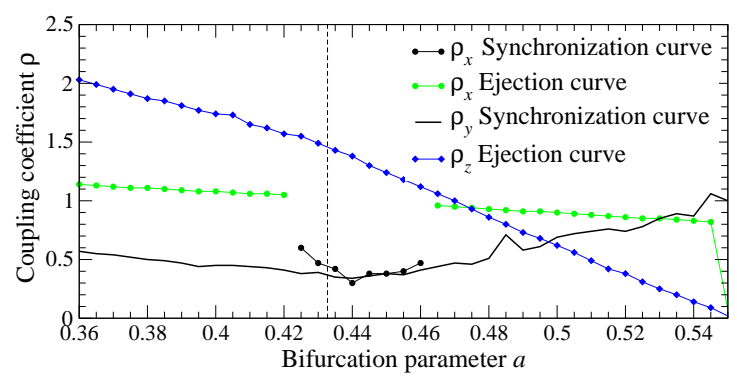

Fig. 1. Critical coupling curves that correspond to the onset of synchronization (synchronization curve) and the ejection of the trajectory to infinity (ejection curve), respectively. Below the synchronization curve, the average distance between points and the first bisecting line of plane $y_{1}-y_{2}$ is greater than 0.1. Case of the Rössler system, $b=2, c=4$ and $\delta \omega=0.04$.

where $\omega$ is used to adjust the pseudo-period of the system. The Rössler systems are coupled according to

$$
\dot{x}_{i}=\boldsymbol{f}\left(\boldsymbol{x}_{i}\right)+k_{p}\left(\boldsymbol{x}_{i-1}-\boldsymbol{x}_{i}\right)+k_{s}\left(\boldsymbol{x}_{i+1}-\boldsymbol{x}_{i}\right)
$$

where $\boldsymbol{x}_{i} \in \mathbb{R}^{3}$ is the state vector of the $i$ th oscillator, and $k_{p}$ and $k_{s}$ two coupling parameters. We choose as a "local oscillator" the Rössler system [6] for three reasons: i) it is not equivariant (this is important since symmetry properties induce quite complex dynamics and tend to reduce the quality of the synchronization), ii) the dynamics of the Rössler system is well known [7], and iii) there is one variable $(y)$ providing a full observability and one variable $(z)$ providing a very bad observability $[8,9]$. The Rössler systems are then either slightly detuned by varying the pseudo-period according to $\delta \omega$ or "perturbed" by varying the value of parameter $a$ using parameter mismatch $\delta a$ as shown in Fig. 2. When the Rössler systems are coupled via variable $y$, all the systems are phase synchronized and have the same dynamics, that is, produce an attractor with the same topology.

When $\delta a$ is decreased from 0 , the chaotic attractor is progressively reduced by pruning the population of unstable periodic orbits up to a simple period-1 limit cycle (Fig. 3). When $\delta a$ is increased, the chaotic behavior is developed as the Rössler system is when parameter $a$ is increased [7]. Around $\delta a=0.15$, the trajectory is ejected to infinity due 


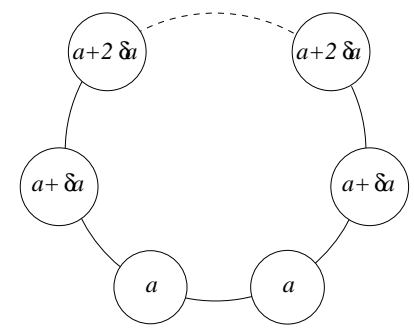

Fig. 2. Parameter mismatch for a ring of an even number of bidirectionally coupled Rössler attractors. The ring has therefore a reflection symmetry (from the parameter point of view).

to a boundary crisis between the attractor and the boundary of the attraction basin as observed in an isolated Rössler system [7]. We did never observe some behaviors which were not solution to the Rössler system.

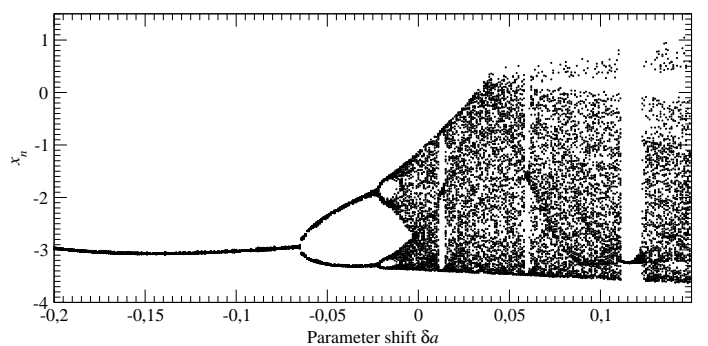

(a) Coupled by variable $y$

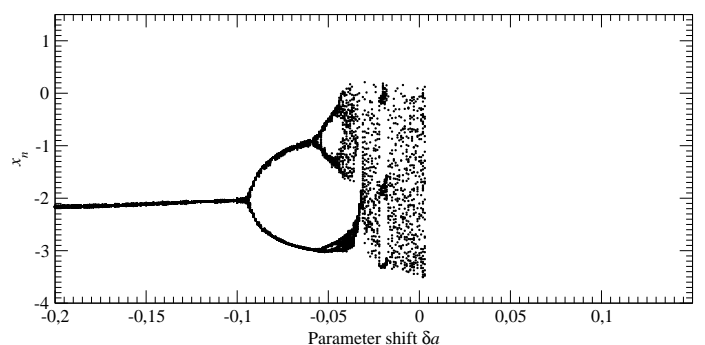

(b) Coupled by variable $x$

Fig. 3. Bifurcation diagram of a local oscillator of a ring of four Rössler systems. Parameter values: $a=0.432, b=2$ and $c=4$.

When the local Rössler systems are coupled using variable $x$ (a variable with a "moderate" observability [4,2]), a reverse bifurcation diagram is also observed (Fig. 3b) for negative parameter mismatch but it is nearly impossible to impose a positive mismatch, the boundary crisis being observed as soon as $\delta a$ is slightly positive. It appears that the lack of observability tends to destabilize the synchronization and, consequently, to develop the local dynamics. All observed behaviors were topologically equivalent to behaviors solution to an isolated Rössler system.

In order to exemplify what occurs when a detuning $\delta \omega$ is introduced between the local oscillators, we choose a ring of six Rössler systems coupled by variable $y$. In that case, the bifurcation diagram versus $\delta \omega$ is rather symmetric with respect to $\delta \omega=0$ with $|\delta \omega|<0.15 \mathrm{~s}$, thus inducing a bubble in the bifurcation diagram as sometines observed in isolated systems (see for instance [10] for bubbling in a forced Duffing system). Increasing $|\delta \omega|$ reduces the development of the chaotic attractor by a sequence of reverse bifurcations, leading to a period-one limit cycle (Fig. 4a). For $|\delta \omega|>0.15 \mathrm{~s}$, there is a departure between negative and positive detunings. When $\delta \omega<0$ (the pseudo-period is thus reduced) the local dynamics is stabilized to a small amplitude limit cycle. Contrary to this, when $\delta \omega>0.15 \mathrm{~s}$, a quasi-periodic regime is observed (Fig. 4b) as exhibited by an annular Poincaré section (Fig. 4c). For the first time, a behavior which was not a solution to the original Rössler system was observed.

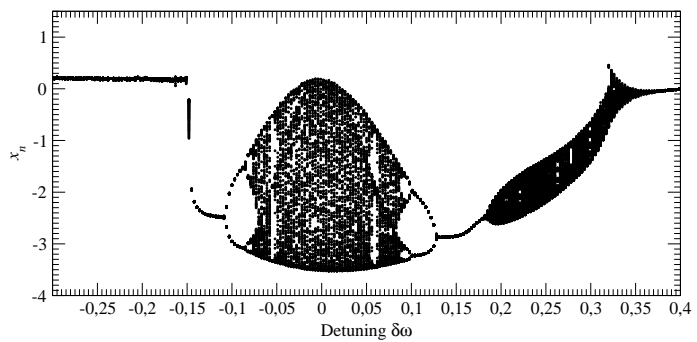

(a) Coupled by variable $y$

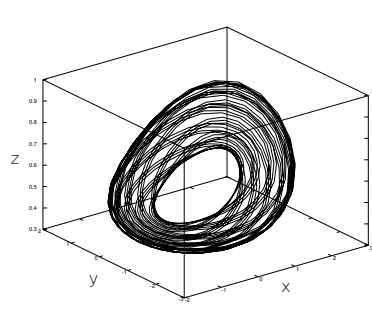

(b) Phase portrait

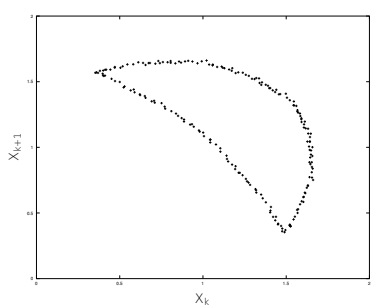

(c) Poincaré section
Fig. 4. Bifurcation diagram of a local oscillator of a ring of six Rössler systems coupled via variable $y$. Parameter values: $a=$ $0.432, b=2$ and $c=4$. The quasi-periodic regime is obtained with detuning $\delta \omega=0.3 \mathrm{~s}$.

\section{References}

1. L. M. Pecora \& T. L. Carroll, Physical Review Letters 64 (1990) 821-824

2. C. Letellier \& L. A. Aguirre, Physical Review E 82 (2010) 016204

3. G. V. Osipov, B. Hu, C. Zhou, M. V. Ivanchenko \& J. Kurths, Physical Review Letters, 91 (2003) 024101

4. C. Letellier, L. A. Aguirre \& J. Maquet, Physical Review E, 71( 2005) 066213

5. C. Letellier \& L. A. Aguirre, Physical Review E 72 (2005) 056202

6. O. E. Rössler, Physics Letters A 57 (1976) 397-398

7. C. Letellier, P. Dutertre \& B. Maheu, Chaos 5 (1995) 271-282

8. C. Letellier, J. Maquet, L. Le Sceller, G. Gouesbet \& L. A. Aguirre, Journal of Physics A 31 (1998) 7913-7927

9. C. Letellier \& L. A. Aguirre, Chaos 12 (2002) 549-558 10. M. Bier \& T. C. Bountis, Physics Letters A, 104 (1984) 239-244 